\title{
Correction to: The Case of Missed Cancers: Applying AI as a Radiologist's Safety Net
}

\author{
Michal Chorev (1), Yoel Shoshan, Ayelet Akselrod-Ballin, \\ Adam Spiro, Shaked Naor, Alon Hazan, Vesna Barros, \\ Iuliana Weinstein, Esma Herzel, Varda Shalev, Michal Guindy, \\ and Michal Rosen-Zvi
}

\section{Correction to:}

Chapter "The Case of Missed Cancers: Applying AI as a Radiologist's Safety Net" in: A. L. Martel et al. (Eds.): Medical Image Computing and Computer Assisted Intervention - MICCAI 2020, LNCS 12266, https://doi.org/10.1007/978-3-030-59725-2_22

The original version of this chapter was revised. Dr. Ayelet Akselrod-Ballin contributed to the development of the conference paper and was therefore added to the list of coauthors. 